\title{
Is Multicentricity Important in Thyroid Papillary Microcarcinomas?
}

\author{
Fatih Ciftci
}

Istanbul Gelisim University, Institute of Health Sciences, Istanbul, Turkey

\section{ABSTRACT}

Aim: The diagnosis of multicentric papillary microcarcinomas of the thyroid gland is often overlooked by preoperative diagnostic methods because of their small size, and the diagnosis is made by histopathological examination of the specimen after surgery. Appropriate surgical treatment should be performed to protect the patient from the risk of secondary surgery. The aim of this study is to determine multicentricity in patients with papillary microcarcinoma of the thyroid and the surgical treatment method that should be applied to these patients.

Materials and Methods: Of 454 patients who underwent thyroid surgery, 163 patients whose histopathology was reported as thyroid cancer were retrospectively evaluated. Of these patients, 113 had papillary microcarcinoma. The frequency of multicentricity of papillary microcarcinoma was investigated in the patient group of which medical records were examined. The patients were staged using MACIS, EORTC, and TNM, which are among the thyroid cancer staging systems.

Results: Multicentricity was found in 26 (23\%) out of 113 papillary microcarcinoma patients. Six (23\%) patients had more than 2 tumor foci. If more than one focus was detected in the same lobe, the incidence of cancer in the other lobe was $31 \%$. Preoperative ultrasonography revealed bilateral multinodular thyroid gland in all patients. Ultrasonography aimed at revealing suspicious findings in terms of malignancy, and $41 \%$ of the patients had these findings. Of the 31 patients who underwent fine-needle aspiration biopsy, 20 (67\%) were evaluated as benign, and 15 (27\%) of 28 papillary microcarcinoma patients who underwent the frozen section procedure were evaluated as benign.

Conclusion: The high rate of multicentricity in papillary microcarcinoma should be considered in the determination of surgical treatment. It is recommended that thyroid ultrasonography should be performed meticulously to avoid multicentricity.

Key words: thyroid, papillary microcarcinoma, multicentricity

\section{INTRODUCTION}

Thyroid cancer is the most common cancer among endocrine cancers $(1,2)$. Approximately $84 \%$ of thyroid cancers are papillary thyroid cancer. "Papillary microcarcinomas," classified as a subgroup of papillary cancers and $\leq 9-10 \mathrm{~mm}$ tumors, account for $30 \%$ of all papillary cancers and are considered to be less aggressive (2-5). Despite high survival rates, local recurrence and metastases occur in some patients and may require more aggressive surgical treatment.

\author{
Corresponding author: \\ Fatih Ciftci, Associatieprofessor M.D \\ Istanbul Gelisim University \\ Insitutue of Health Sciences \\ Avcilar, Istanbul, Turkey \\ Tel: 905056164248 \\ Fax: 902124086603 \\ E-mail: oprdrfatihciftci@gmail.com
}

Received: 30.10.2019

Accepted: 02.12.2019 
Although there is some confusion about its definition in the literature, "multicentricity in thyroid cancer" is defined as the presence of thyroid cancer in the same thyroid lobe or in more than one location within two lobes (6).

It is not easy to determine the diagnosis and multicentric feature of papillary microcarcinoma by the commonly used diagnostic methods nowadays. Microcarcinomas are generally non-palpable and clinically silent. The incidence of papillary microcarcinoma in autopsy studies varies locally between $0.01 \%$ and $35 \%$, although these rates support the fact that most papillary microcarcinomas are benign $(2,3,7,8)$. Most microcarcinoma diagnoses result from the pathological examination of the specimen of the patient who has been operated on for "incidentally" benign thyroid disease. Nowadays, the diagnosis of papillary microcarcinoma has increased thanks to the high-resolution ultrasonography and fine-needle aspiration biopsy applied to nodules smaller than $1 \mathrm{~cm}$ in diameter, which are suspected in ultrasonography. In this clinical study, we aimed to determine the frequency, multicentricity rates and optimal treatment approach of patients with thyroid papillary microcarcinoma.

\section{MATERIALS AND METHODS}

In our study, 163 patients with thyroid cancer among 454 patients who had undergone thyroid surgery between July 2009 and November 2018 were analyzed retrospectively. A total of 113 (69.3\%) papillary microcarcinoma cases were identified. Patients were examined for age, sex, and MACIS (Metastases, Age, Completeness of Resection, Invasion, Size)(8), TNM (9) and EORTC (European Organization for Research and Treatment of Cancer)(10) staging systems.

In daily practice, all patients undergo neck ultrasonography (US) before making the decision on an operation. However, the thyroid volume related to thyroid parenchyma (homogenous, non-homogenous, with multiple lesions); nodule echogenicity (hyper-iso or hypoechoic) nodule structure (solid, mixed or cystic); nodule boundaries (well-defined, irregular or unclear); the presence of hyperechogenic stains (coarse or microcalcifications), and finally, the blood supply pattern of the nodule are the common parameters evaluated. US-guided fine-needle aspiration biopsy (FNAB) is performed in patients who are suspected ultrasonographically after US evaluation. FNAB results are classified as benign, suspicious, malignant, or inadequate, and samples showing cellular atypia or suggesting follicular lesions are included in the suspicious group. When FNAB is reported as malignant, the cancer subtype cannot be differentiated. All patients undergo thyroid hormone screening and indirect laryngoscopy before surgery. Routine surgical methods used in our clinic for multinodular disease are near-total thyroidectomy (leaving less than $2 \mathrm{~g}$ thyroid tissue when necessary to protect the parathyroid or recurrent laryngeal nerve) or total thyroidectomy according to the results of intraoperative exploration and preoperative examinations. During the operation, both lobes and isthmus are carefully evaluated for the presence of any disease. SPSS (version 16.0) statistical software was used for computerized data analysis.

\section{RESULTS}

The mean age of the patients was $46 \pm 10.9$, years and the age range was $18-75$, while $89(78.7 \%)$ of the patients were female, and $24(21.3 \%)$ were male. The preoperative US revealed bilateral multinodular thyroid gland in all patients. Retrospective findings that were suspected for malignancyin the US were examined,and $47(41.5 \%)$ of the patients had these findings (Table 1 ).

Multicentricity was detected in $26(23 \%)$ of 113 patients with papillary microcarcinoma. Six (23\%) patients had more than two tumor foci. In $31 \%$ of the cases, the second tumor focus was in the same lobe, whereas in $69 \%$, it was in the opposite lobe. If more than one focus was detected in the same lobe, the

Table 1 - The patient that Suspect sign in the ultrasonography

\begin{tabular}{|c|c|c|c|}
\hline Ultrasonoraphy finding in nodule & Patient number & Gender & Patient number (n/\%) \\
\hline Border irregularity & $1(2.1 \%)$ & Female & $89(78.7 \%)$ \\
\hline microcalcifications & $7(14.8 \%)$ & Male & $24(21.3 \%)$ \\
\hline Solid nodule & $11(47 \%)$ & & \\
\hline Cutting of hypoecoic halo & $1(2.1 \%)$ & & \\
\hline Central vascularization & $1(2.1 \%)$ & & \\
\hline Hypoechogenic nodul & $15(31.9 \%)$ & & \\
\hline
\end{tabular}


incidence of cancer in the other lobe was $34 \%$. Of the 113 patients, 31 patients underwent FNAB, and biopsy results of $21(67 \%)$ patients were reported to be "benign". The biopsy of 16 patients with FNAB results reported as benign was performed in the external center and re-evaluated in our center. In 11 patients, from which nodule FNAB was performed could not be determined. The biopsy was performed in two patients in the thyroid lobe without cancer, while it was performed in five patientsin the dominant nodule in the lobe with cancer. Only 2 of the 13 FNABs performed under US guidance in our centerwere reported to be "benign." Total thyroidectomy was performed in 95 (84\%) patients, and near-total thyroidectomy was performed in $18(16 \%)$ patients (Table 2).

Among the patients who underwent frozen section examination during the surgery and whose postoperative pathologies were reported as papillary microcarcinoma, frozen section examination results were reported as "benign" in 8 (28.5\%), "malignant" in 19 $(67.8 \%)$ and "suspicious" in 1 (3.5\%). One of the patients who underwent thyroid surgery also underwent simultaneous lymph node dissection. It was determined that two patients had thyroid capsule invasion and one patient had lymph node metastasis (left lateral + central neck dissection was performed). Eighteen patients with multicentric and/or lymph node metastases underwent radioactive iodine ablation treatment. All patients were included in Group 1 and Stage 1 according to MACIS and TNM classifications, whereas $92 \%$ of the patients were classified as Group 1 according to EORTC. The distribution of tumors according to diameters is presented in Table 3 .

\section{DISCUSSION}

Multicentricity in thyroid papillary microcarcinomas is defined as the presence of cancers in the same thyroid lobe or in more than one location within two lobes. The diagnosis of papillary microcarcinoma and determination of its multicentricity are difficult in the preoperative period. In our study, the rate of multicentricity was found to be $23 \%$, while the risk of cancer in the opposite lobe was $32 \%$ in patients with multiple foci in the same lobe.

The diagnosis of multicentric microcarcinomas is often overlooked with preoperative diagnostic methods due to their small size, and diagnosis is made by pathological examination of the specimen after surgery $(10,11)$. In this case, appropriate surgical treatment should be applied to protect the patient from the risk of secondary surgery, and it should be
Table 2 - Treatment of the papillary microcarcinoma

\begin{tabular}{lc}
\hline Treatment & Patient number (n/\%) \\
\hline Thyroidectomy & 113 \\
\hline Total & $95(84 \%)$ \\
\hline Nearthe total & $18(16 \%)$ \\
\hline Radioactive iode treatment & 113 \\
\hline Yes & $18(15.9 \%)$ \\
\hdashline No & $95(84.0)$ \\
\hline Dissection of lymph nodules & $2(1.5 \%)$ \\
\hline
\end{tabular}

carefully evaluated by preoperative ultrasonography (US). Criteria such as marginal irregularity, the presence of microcalcification, marked hypoechogenicity, the presence of central blood supply, and hypoechoic halo disruption in any nodule in ultrasonography are predictive of malignancy potential. In ultrasonography, papillary carcinomas usually appear as hypoechoic nodules and often have solid characteristics. Intralesional punctate calcifications are characteristic of $85-90 \%$ of these tumors and are a reliable finding for the sonographic diagnosis of papillary carcinomas (12). When a nodule is detected by ultrasonography, the main problem is to decide whether it is benign or malignant. For this purpose, all sonographic findings described in Table 1 should be analyzed separately and then combined with clinical findings to differentiate benign nodules and malignant nodules (FNAB for cytological examination should be performed if malignancy is suspected). It should be kept in mind that complementary thyroidectomy and lymph node dissections are not completely risk-free.In our study, false-negative rates of FNAB and frozen section applications were $69 \%$ and $29 \%$, respectively. The false-negative rate of FNAB was reported to be $4 \%-13 \%$ in the studies conducted (13). We believe that the high false-negative rate in FNAB results is due to the fact that the nodule from which FNAB was taken could not be determined because FNAB was performed in the external center in 13 of the 31 patientsand the biopsy was performed in the non-cancerous thyroid lobe in 2 patients. The false-negative rate in FNABs performed in our center

Table 3 - Tumor size in papillary microcarcinoma

\begin{tabular}{cc}
\hline Tumor size $(\mathrm{mm})$ & $\mathbf{N}(\%)$ \\
\hline$\leq 1$ & $15(13.2)$ \\
\hline $2-4$ & $36(31.8)$ \\
\hline $5-7$ & $39(34.5)$ \\
$8-10$ & $23(20.3 \%)$ \\
\hline
\end{tabular}


was $13 \%$. Of the 17 patients who underwent frozen section examination and whose results were reported as benign, malignant nodules were in the other lobe in 4 patients and in the same lobe in 13 patients. In order to show that preoperative and peroperative diagnostic methods may be insufficient for the diagnosis of papillary microcarcinoma and multicentricity and frozen section examination will not provide an additional benefit in the management of the treatment due to high false-negative rates, many classifications have been made for thyroid cancers. MACIS, TNM and EORTC classification systems were used for patient staging. Tumor size and peripheral invasion, lymph node involvement, metastasis parameters are used in TNM classification; metastasis, age, the adequacy of resection, invasion, size parameters are used in MACIS classification; age, gender, histologic type, the presence of anaplastic carcinoma, tumor burden and metastatic foci parameters are used in EORTC classification. While MACIS is specific for papillary tumors, TNM and EORTC are for all tumors. By using these classification systems, it was determined which patients belong to low or high-risk groups. The risk level of the patient is an important factor in determining the treatment method to be applied.

Although survival rates are reported to be near perfect, the potential for metastasis and high multicentricity of papillary microcarcinomas should be kept in mind. When total or near-total thyroidectomy is performed in multicentric papillary carcinoma, the probability of recurrence is $5 \%$ since all foci are removed and $20 \%$ in lobectomy patients (14). Therefore, we think that ultrasonography should be performed meticulously in preoperative evaluation in order to avoid multicentricity.

It was observed that papillary microcarcinomas, of which frequency increases in the process of change in thyroid cancer, constitute about one-third of all thyroid cancers. Nowadays, the frequency of diagnosis of papillary microcarcinomas has increased after US started to be widely used in neck pathologies. Multicentricity was detected in 33 (24\%) out of 113 papillary microcarcinoma patients. Studies have shown that the rate of multicentricity in papillary microcarcinoma varies between $9.5 \%$ and $24.9 \%(15,16,17)$. Cytological examination should be definitely performed in case of suspicion of malignancy in US, i.e. marginal irregularity in the nodule, the presence of microcalcification, marked hypoechogenicity, the presence of central blood supply, hypoechoic halo disruption and solid nodule, and a surgical treatment method should be determined by evaluating cytology results and other laboratory tests and clinical findings together.

\section{CONCLUSION}

The high rate of multicentricity in papillary microcarcinoma should be taken into consideration during the determination of surgical treatment. Therefore, FNAB should be performed from a nodule that is suspected ultrasonographically instead of a dominant nodule. In patients with multinodular goiter, total or near-total thyroidectomy should be preferred instead of frozen section examination because the false-negative rate of frozen section examination may be high.

Conflict of interest: None declareted.

Source and Support: Nil.

\section{REFERENCES}

1. Kaliszewski K, Zubkiewics-Kucharska A, Kielb P, Maksymowicz J, Krawczyk A, Krawiec 0. Comparison of the prevalence of incidental and non-incidental papillary thyroid microcarcinoma during 20082016: a single-center experience. World J surg oncol.2018; 16(1):202-208.

2. Meilinger-Dobra M, Remenar E, Fröhlich G, Sinkovics I, Peter I, Boer A. Retrospective analysis of papillary thyroid microcarcinoma cases treated between 2001 and 2010 in the Hungarian National Institute of Oncology. Magy Onkol. 2018; 62(3):153-158.

3. Price AK, Randle RW, Schneider DF, Sippel RS, Pitt SC. Papillary thyroid microcarcinoma: decision-making, extent of surgery, and outcomes. J Surg Res. 2017; 218:237-245

4. Gui CY, Qui SL, Peng ZH, Wang M. Clinical and pathologic predictors of central lymph node metastasis in papillary thyroid microcarcinoma: a retrospectivecohortstudy. J Endocrinol Invest. 2018;41(4):403-409.

5. Takano T. Natural history of thyroidcancer. Endocr J. 2017; 63(3):237-244

6. Liu LS, Liang J, Li JH, Liu X, Jiang L, Long JX, et al. The incidence and risc factors for central lymph node metastasis in cNO papillary thyroid microcarcinoma: a meta-analysis. Eur Arch Otorhinolaryngol. 2017;274(3):1327-1338.

7. Miyauchi A. Clinical trials of active Surveillance of papillary microcarcinoma of the thyroid. World J surg. 2016;40(3):516-522.

8. Alhashemi A, Goldstein DP, Sawka AM. A systematic review of primary active surveillance management of low-risk papillary carcinoma. Curr Opin Oncol. 2016;28(1):11-7.

9. Huang SH, O'Sullivan B. Overview of the 8th Edition TNM Classification for Head and Neck Cancer. Curr Treat Options Oncol. 2017;18(7):40

10. Byar DP, Green SB, Dor P, Williams ED, Colon J, vanGilse HA, et al. A prognostic index for thyoid carcinoma. A study of the E.O.R.T.C. Thyroid Cancer Cooperative Group. Eur J Cancer 1979;15:1033-1041

11. Gschwandtner E, Klatte T, Swietek N, Bures C, Kober F, Ott J, et al. 
Increase of Papillary Thyroid Microcarcinoma and a Plea for Restrictive Treatment: A Retrospective Study of 1,391 Prospective Documented Patients. Surgery. 2016;159(2):503-11.

12. Qu N, Zhang L, Ji QH, Chen JY, Zhu YX, Cao YM, et al. Risk factors for central compartment lymph node metastasis in papillary thyroid microcarcinoma: A Meta-Analysis. World J Surg. 2015;39(10): 2459-70.

13. Cho SY, Lee TH, Ku YH, Kim HI, Lee GH, Kim MJ. Central lymph node metastasis in papillary thyroid microcarcinomas can be stratified according to the number, the size of metastatic foci, and the presence of desmoplasia. Surgery. 2015;157(1):111-8.

14. Wang TS, Goffredo P, Sosa JA, Roman SA. Papillary thyroid microcarcinoma: an over-treated malignancy? World J Surg. 2014;38(9):
2297-303.

15. Lee HS, Park HS, Kim SW, Choi G, Park HS, Hong JC, et al. Clinical characteristics of papillary thyroid microcarcinoma less than or equal to $5 \mathrm{~mm}$ on ultrasonography. Eur Arch Otorhinolaryngol. 2013;270(11):2969-74.

16. Vasileiadis I, Karatzas T, Vasileiadis D, Kapetakanis S, Charitoudis G, Karakostas E, et al. Clinical and pathological characteristics of incidental and nonincidental papillary thyroid microcarcinoma in 339 patients. Head Neck. 2014;36(4):564-70

17. Karatzas T, Vasiliiadis I, Kapetakanis S, Karakostas E, Chrousos G, Kouraklis $\mathrm{G}$. Risk factors contributing to the difference in prognosis for papillary versus micropapillary thyroid carcinoma. Am J Surg. 2013;206(4):586-93. 